\title{
Association of TCF4 and CLU polymorphisms with Fuchs' endothelial dystrophy and implication of CLU and TGFBI proteins in the disease process
}

\author{
Abraham Kuot $^{1}$, Alex W Hewitt ${ }^{2}$, Kim Griggs ${ }^{3}$, Sonja Klebe ${ }^{3}$, Richard Mills ${ }^{1}$, Vishal Jhanji ${ }^{2,4}$, Jamie E Craig ${ }^{1}$, \\ Shiwani Sharma ${ }^{1,5}$ and Kathryn P Burdon ${ }^{\star, 1,5}$
}

Fuchs' endothelial dystrophy (FED) is a disease affecting the corneal endothelium. Recent studies reported significant association of polymorphisms in the TCF4 (transcription factor 4) gene, and a borderline association of PTPRG (protein tyrosine phosphatase, receptor type, G) variants with late-onset FED in Caucasians from the United States. Association of TCF4 has also been reported in the Chinese population. We aimed to determine association of the reported polymorphisms in TCF4 and PTPRG, and association of polymorphisms in the candidate genes ZEB1 (zinc-finger E-box binding homoebox 1), COL8A2 (collagen, type VIII, alpha 2), TGFBI (transforming growth factor, $\beta$-induced) and CLU (clusterin) in Australian cases. We also compared the expression of TGFBI and CLU proteins between FED and normal whole corneas. In all, 30 single-nucleotide polymorphisms (SNPs) from the candidate genes were genotyped in 103 cases and 275 controls. Each SNP and haplotype was assessed for association with the disease. SNP analysis identified an association of $T C F 4$ ( $r 613872\left(P=5.25 \times 10^{-15}\right.$, $\mathrm{OR}=4.05)$, rs9954153 $\left(P=3.37 \times 10^{-7}, \mathrm{OR}=2.58\right), \mathrm{rs} 2286812\left(P=4.23 \times 10^{-6}, \mathrm{OR}=2.55\right)$ and $\mathrm{rs} 17595731\left(P=3.57 \times 10^{-5}\right.$, $\mathrm{OR}=3.79)$ ), $C L U(\mathrm{rs} 17466684 ; P=0.003, \mathrm{OR}=1.85)$ and one haplotype of $T G F B I \mathrm{SNPs}(P=0.011, O R=2.29)$ with FED in Caucasian Australians. No evidence for genetic association of PTPRG, ZEB1 and COL8A2 was found. Immunohistochemistry showed differential expression of CLU and TGFBI proteins in FED-affected compared with normal corneas. In conclusion, variation in TCF4, CLU and TGFBI, but not PTPRG, ZEB1 and COL8A2 genes are associated with FED in Caucasian Australian cases. Differential expression of CLU and TGFBI proteins in FED-affected corneas provides novel insights into the disease mechanism.

European Journal of Human Genetics (2012) 20, 632-638; doi:10.1038/ejhg.2011.248; published online 11 January 2012

Keywords: cornea; corneal dystrophy; eye; immunohistochemistry; protein expression

\section{INTRODUCTION}

Fuchs' endothelial dystrophy (FED, MIM 136800) is a degenerative disease of the corneal endothelium (CE). ${ }^{1}$ The earliest clinical feature of FED is progressive formation of microscopic outgrowths (guttae) in Decemet's Membrane (DM), ${ }^{2}$ a collagen-rich basal lamina secreted by the CE. ${ }^{3}$ Early stages of FED are characterised by aberrant thickening of DM and gradual loss of the non-regenerative CE. ${ }^{4}$ This impairs the ability of the endothelium to pump excess fluid out of the corneal stroma, ${ }^{1}$ resulting in corneal oedema, and ultimately leading to vision loss and pain. ${ }^{2}$ Corneal transplantation using post-mortem donor tissue is the only effective treatment for FED. ${ }^{5}$

The prevalence of FED varies markedly across the world. It affects $\sim 4 \%$ of the USA population over the age of 40 years, ${ }^{6}$ but is less frequent in Asian ${ }^{7}$ and Middle-Eastern populations. ${ }^{8}$ In Australia, corneal grafting for FED accounts for $\sim 6 \%$ of all corneal grafts performed annually. ${ }^{9}$

FED is a genetically heterogeneous disease. There are two forms defined by the age of onset. Early-onset FED is rare ${ }^{10}$ and is typically inherited as an autosomal dominant disease with high penetrance and almost uniform expressivity. ${ }^{2}$ Mutations in the COL8A2 (collagen, type
VIII, alpha 2) gene cause some cases of early-onset FED. ${ }^{11}$ COL8A2 is an extracellular matrix protein and is a major component of DM. ${ }^{12}$ The more common late-onset FED can either be familial or sporadic, with onset typically after the age of 40 years. ${ }^{13}$ The risk of developing the late-onset form increases with age and female sex. ${ }^{6}$ Familial lateonset FED shows an autosomal dominant inheritance with high penetrance, but variable expressivity. ${ }^{6}$ Mutations in the ZEB1 (zinc-finger E-box binding homoebox 1) gene can cause both sporadic and familial late-onset FED. ${ }^{6,14}$ ZEB1 is expressed in the CE. ${ }^{15}$ It regulates cell proliferation and differentiation by inducing epithelial-mesenchymal transition. ${ }^{16,17}$ The SLC4A11 (solute carrier family 4, sodium borate transporter, member 11) mutations cause sporadic and familial late-onset FED. ${ }^{8,18}$ Mutations identified in the COL8A2, ZEB1 and SLC4A11 genes account for a small number of FED cases. Furthermore, four chromosomal loci are associated with late-onset FED, but the causative genes remain to be identified. ${ }^{19}$

Recently, single-nucleotide polymorphisms (SNPs) in the TCF4 (transcription factor 4) gene, encoding the E2-2 protein, were reported to be significantly associated with late-onset FED in Caucasian

${ }^{1}$ Department of Ophthalmology, Flinders University, Bedford Park, South Australia, Australia; ${ }^{2}$ Centre for Eye Research Australia, Royal Victorian Eye and Ear Hospital, East Melbourne, Victoria, Australia; ${ }^{3}$ Department of Anatomical Pathology, Flinders University, Bedford Park, South Australia, Australia; ${ }^{4}$ Department of Ophthalmology and Visual Sciences, The Chinese University of Hong Kong, Hong Kong

*Correspondence: Dr KP Burdon, Department of Ophthalmology, Flinders University, Bedford Park, South Australia 5042 , Australia. Tel: +61 8 8204 4094 ; Fax: +61 88277 0899; E-mail: kathryn.burdon@flinders.edu.au

${ }^{5}$ These authors contributed equally to this work.

Received 27 July 2011; revised 8 November 2011; accepted 25 November 2011; published online 11 January 2012 
Americans. ${ }^{19}$ In the same study, SNPs in the PTPRG (protein tyrosine phosphatase, receptor type, $G$ ) gene were nominally associated with FED, but the association did not reach genome-wide significance. Independent replication studies have confirmed association of TCF4 variants with late-onset FED in Caucasian American ${ }^{20}$ and Chinese cohorts, ${ }^{21}$ but the association with PTPRG remains to be confirmed.

Proteomic analyses have shown overexpression of TGFBI (transforming growth factor, $\beta$-induced) and CLU (clusterin) proteins in the CE+DM complex from FED patients compared with normal controls. ${ }^{22} \mathrm{CLU}$ is a molecular chaperone and is implicated in protecting cells from effects of physiological stress caused by aging, oxidative stress and apoptosis, which are proposed as potential mechanisms underlying FED pathophysiology. ${ }^{23-25}$ TGFBI is a secreted extracellular matrix protein that mediates cell adhesion by interacting with major protein components of DM, including collagens. ${ }^{22}$ FED is characterised by breakdown of tight junctions between corneal endothelial cells and transformation into mesenchymal cells, a feature of aberrant cell adhesion. TGFBI is induced by the TGF- $\beta$ (transforming growth factor- $\beta$ ) gene. ${ }^{26}$ Interestingly, TGF- $\beta$ reportedly regulates both TCF4 and ZEB1, which have been implicated in FED. ${ }^{27}$

This study investigated association of the reported polymorphisms in the TCF4 and PTPRG genes in Australian FED cases. It also investigated association of SNPs in the candidate genes ZEB1, COL8A2, TGFBI and CLU in these cases. The CLU and TGFBI genes were chosen because their function relates to the pathophysiology of FED and the encoded proteins are overexpressed in the diseased DM. FED-associated mutations in SLC4A11 were reported in four FEDaffected Asian cases ${ }^{8}$ at the time of this study. However, no diseaseassociated mutations have been reported in Caucasians; therefore, association of this gene was not assessed. We also performed immunohistochemistry to compare expression and distribution of the TGFBI and CLU proteins in sections of FED and normal whole corneas. Our data support genetic association of the TCF4 gene with FED in Caucasian Australians and show novel but nominal association of $C L U$ with FED in these cases. Furthermore, these data show that TGFBI and CLU are expressed in the corneal epithelium of FED corneas, but not in control corneas, also a novel finding.

\section{MATERIALS AND METHODS}

\section{Participant recruitment and sample collection}

This study was approved by the Southern Adelaide Health Service/Flinders University Clinical Research Ethics Committee and the Royal Victorian Eye and Ear Hospital Human Research Ethics Committee, in accordance with the requirements of the National Health and Medical Research Council Statement on Ethical Conduct in Human Research (2007). Patients diagnosed with Grade 3-6 advanced $\mathrm{FED}^{28}$ were recruited and gave written informed consent. Blood samples were collected from 103 participants through the Flinders Eye Clinic (Adelaide, SA, Australia) and the Royal Victorian Eye and Ear Hospital (Melbourne, VIC, Australia). Genomic DNA was extracted using QIAamp DNA Blood Maxi kit (Qiagen Pty Ltd, Doncaster, VIC, Australia), following the manufacturer's protocol. Control genomic DNA samples were from unrelated, unaffected South Australian residents aged over 50 years recruited previously for use as controls in a variety of ocular genetic studies. Two normal and three FED corneas for immunohistochemistry were provided by the Department of Anatomical Pathology (Flinders University, Bedford Park, SA, Australia). FED corneas were obtained for histopathological purposes following corneal transplantation, whereas normal corneas were from deceased donors deemed unsuitable for corneal transplantation and obtained through the Eye Bank of South Australia.

Genotyping and data analysis

FED-associated SNPs in TCF4 (rs613872, rs17595731, rs2286812 and rs9954153) and PTPRG (rs7640737 and rs10490775) were selected from the literature ${ }^{19}$ for replication in this study. Tag SNPs in the ZEB1, COL8A2, TGFBI and $C L U$ genes were selected on the basis of linkage disequilibrium patterns observed in the Caucasian samples genotyped as part of the International HapMap Project (http://hapmap.ncbi.nlm.nih.gov/) using the tagger program implemented in Haploview. ${ }^{29}$ Only SNPs conforming to Hardy-Weinberg equilibrium, having no Mendelian genotyping errors and a minimum minor allele frequency of $10 \%$, were included. The linkage disequilibrium $\left(r^{2}\right)$ threshold was set to 0.8. All SNPs were genotyped in 103 FED cases and 275 normal controls using iPlex Gold chemistry (Sequenom Inc., Herston, QLD, Australia) on a MassArray Spectrophotometer (Sequenom Inc.) at the Australian Genome Research Facility (Brisbane, QLD, Australia). Genotypes were analysed using PLINK (http://pngu.mgh.harvard.edu/ purcell/plilnk/ dataman.shtml). ${ }^{30}$ Chi-square $\left(\chi^{2}\right)$ and/or Fisher's exact tests were conducted to assess inheritance models. Significantly associated SNPs were analysed using logistic regression in PLINK to explore the effects of age and sex, although age of recruitment was only available for 258 controls. Analysis of haplotypes with $>1 \%$ frequency was also performed using PLINK. $P$-values and odds ratios (ORs) were calculated for each haplotype compared with all others. Power calculations were conducted using the Genetic Power Calculator (http://pngu.mgh.harvard.edu/ purcell/gpc). ${ }^{31}$

\section{Immunohistochemistry}

Paraffin-embedded sections of FED and normal corneas were immunolabelled for the CLU and TGFBI proteins as described previously, ${ }^{32}$ except for the following variations.

Citric acid antigen retrieval $(0.011 \mathrm{~m}$ sodium citrate, $\mathrm{pH}$ 6) was performed for immunolabelling with the anti-TGFBI antibody, and alkaline antigen retrieval $(1 \times$ Dako Target Retrieval Solution, $\mathrm{pH}$ 9; Dako Australia Pty Ltd, Scoresby, VIC, Australia) for labelling with the anti-clusterin antibody. Slides were placed in retrieval buffer and heated to boiling point for $4 \mathrm{~min}$ in a microwave, before resetting the microwave to low mode and heating the slides for further $10 \mathrm{~min}$. Sections were cooled for $1 \mathrm{~h}$ at room temperature, and washed in TBS before incubation with rabbit polyclonal anti-human TGFBI antibody (1:1000; HPA008612; Sigma-Aldrich Inc., St Louis, MO, USA) or anti-human clusterin antibody (1:2000; Sc-8354; Santa Cruz Biotechnology, Santa Cruz, CA, USA) at $4^{\circ} \mathrm{C}$ overnight. The sections were incubated for $1 \mathrm{~h}$ at room temperature with the NovoLink Polymer complex reagent (Leica Microsystems, Bannockburn, IL, USA). Primary antibody binding was detected with Liquid DAB+ Substrate Chromogen System (K3468; Dako Australia Pty Ltd). Sections were counterstained with haematoxylin and mounted in DePeX (Merck KGaA, Darmstadt, Germany). Light microscopy and photography were performed on an Olympus BX41 microscope fitted with a digital DP20/DP70 camera using CellSens Standard Photography software (Olympus Corporation, Tokyo, Japan).

\section{RESULTS}

\section{Genetic analyses}

Genotypes of the chosen SNPs were obtained for 103 FED cases and 275 unaffected controls. The mean sex and age distribution of cases and controls is provided in Table 1 . Controls were significantly older than cases by design to reduce the likelihood of controls having yet to manifest the disease. The case cohort had a higher percentage of females than the controls consistent with the reported higher prevalence of the disease in females. ${ }^{6}$ This study had $80 \%$ power to detect a moderate effect size of genotypic relative risk of 1.5 under an

\section{Table 1 Characteristics of the study population}

\begin{tabular}{lccc}
\hline Variables & Cases & Controls & P-value \\
\hline Total number of participants & 103 & 275 & \\
Total number of female participants (\%) & $68(68 \%)$ & $138(53 \%)$ & 0.01 \\
Average age (SD) (in years) & $68.6(15.5)$ & $76.0(8.4)$ & $<0.001$
\end{tabular}

Total number of participants with case and control status is given along with sex and age distributions. 
additive model, assuming a disease prevalence of $4 \%$ and a risk allele frequency of $20 \% .^{31}$

Allele frequencies and association results for genotyped SNPs are given in Table 2. We detected significant allelic association between FED and each SNP from the TCF4 locus. The G allele of the TCF4 intronic SNP rs613872 showed the most significant association with FED $\left(P=5.25 \times 10^{-15}\right.$; OR $\left.(95 \% \mathrm{CI})=4.05(2.82-5.83)\right)$. Genotypic analysis indicated significant association of the four TCF4 SNPs under the dominant model $\left(P=4.54 \times 10^{-16}\right.$ at rs613872; $P=4.39 \times 10^{-7}$ at rs9954153; $P=8.65 \times 10^{-6}$ at $\mathrm{rs} 2286812$ and $P=4.05 \times 10^{-5}$ at rs17595731). These results replicate the findings of previous studies in Caucasians. ${ }^{19,20}$ The rs 10490775 and rs7640737 SNPs from the PTPRG locus showed a borderline allelic association with FED in this study.

Of the seven tag SNPs from CLU, rs17466684 showed significant allelic association with FED $(P=0.003$; OR $(95 \% \mathrm{CI})=1.85(1.23-2.78))$, (Table 2) under a dominant model $(P=0.006)$. Logistic regression data revealed that rs 17466684 is associated with FED independently of age and sex in this cohort $(P$-value $=0.0056$, OR $(95 \% \quad \mathrm{CI})=1.86$ $(1.20-2.87))$. Haplotype analyses of the genotyped SNPs in $C L U$ revealed significant association with FED with an overall $P$-value of 0.049 (Table 3). Three specific haplotypes were associated.
Table 3 Association between common haplotypes of tag SNPs in the $C L U$ gene and FED

\begin{tabular}{lcccc}
\hline & \multicolumn{4}{c}{ Overall P-value $=0.049$} \\
Haplotype & $f$ cases & $f$ controls & P-value & OR $(95 \% \mathrm{Cl})$ \\
\hline GAGTCGA & 0.30 & 0.30 & 0.804 & $0.95(0.65-1.40)$ \\
GAGCGGA & 0.17 & 0.17 & 0.992 & $1.00(0.67-1.49)$ \\
GGACCCA & 0.02 & 0.04 & $\mathbf{0 . 0 3 5}$ & $\mathbf{0 . 2 7}(0.06-1.10)$ \\
AAACCCG & 0.06 & 0.02 & $\mathbf{0 . 0 1 7}$ & $\mathbf{3 . 1 1}(\mathbf{1 . 2 2}-7.97)$ \\
AAACCCA & 0.01 & 0.01 & 0.748 & $0.75(0.13-4.49)$ \\
GAGTCGG & 0.08 & 0.08 & 0.665 & $0.88(0.48-1.60)$ \\
AAGTCGA & 0.04 & 0.01 & $\mathbf{0 . 0 1 1}$ & $\mathbf{5 . 2 0}(1.43-19.0)$ \\
GAACCCG & 0.05 & 0.03 & 0.463 & $1.41(0.57-3.45)$ \\
GAACCCA & 0.02 & 0.03 & 0.282 & $0.51(0.14-1.86)$ \\
GAACCGA & 0.04 & 0.03 & 0.672 & $1.21(0.50-2.92)$ \\
GAGCGGG & 0.10 & 0.14 & 0.148 & $0.67(0.39-1.17)$ \\
AGACCCA & 0.06 & 0.05 & 0.565 & $1.28(0.56-2.91)$ \\
AGACCCG & 0.06 & 0.04 & 0.369 & $1.38(0.69-2.77)$ \\
GGACCCG & 0.01 & 0.03 & 0.068 & $0.26(0.05-1.36)$
\end{tabular}

Abbreviations: $f$, frequency; $\mathrm{OR}$, odds ratio; $\mathrm{Cl}$, confidence interval.

Significant associations are in bold font.

Table 2 Results of association tests for SNPs from TCF4 and PTPRG, and tag SNPs from ZEB1, COL8A2, TGFBI and CLU loci with FED

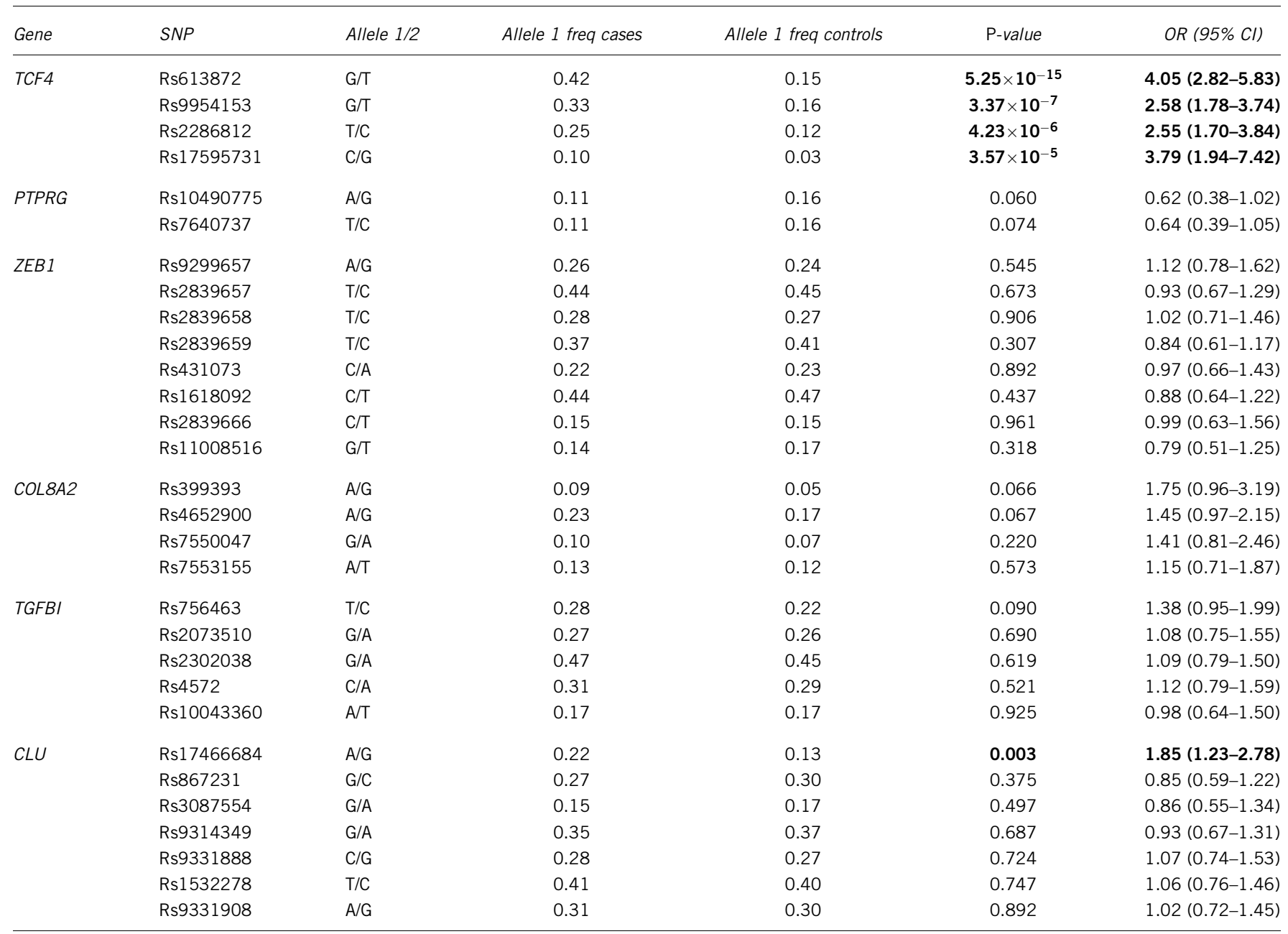


Table 4 Association between common haplotypes of tag SNPs in the TGFBI gene and FED

\begin{tabular}{lcccc}
\hline & \multicolumn{4}{c}{ Overall P-value $=0.057$} \\
Haplotype & $f$ cases & $f$ controls & P-value & OR $(95 \% \mathrm{Cl})$ \\
\hline TAAAT & 0.11 & 0.05 & $\mathbf{0 . 0 1 1}$ & $\mathbf{2 . 2 9}(\mathbf{1 . 2 3 - 4 . 2 8 )}$ \\
CAAAT & 0.44 & 0.51 & 0.083 & $0.75(0.54-1.04)$ \\
CGGCT & 0.29 & 0.27 & 0.622 & $1.09(0.77-1.55)$ \\
TAGAA & 0.17 & 0.17 & 0.984 & $1.00(0.64-1.56)$ \\
\hline
\end{tabular}

Abbreviations: $\mathrm{f}$, frequency; OR, odds ratio; $\mathrm{Cl}$, confidence interval.

Significant associations are in bold font.

The GGACCCA haplotype appears to be protective $(P=0.035$; OR $(95 \% \mathrm{CI})=0.27(0.06-1.10))$, while AAACCCG $(P=0.017$; OR $(95 \%$ $\mathrm{CI})=3.11(1.22-7.97))$ and AAGTCGA $(P=0.011$; OR $(95 \% \mathrm{CI})=5.20$ (1.43-19)) haplotypes increase the risk of FED. Tag SNPs in the ZEB1, COL8A2 and TGFBI genes revealed no allelic association with FED (Table 2). However, haplotype analyses of SNPs in the TGFBI gene revealed a borderline association (overall $P$-value $=0.057$; Table 4 ). The TAAAT haplotype showed significant association $(P=0.011$; OR $(95 \%$ $\mathrm{CI})=2.29(1.22-7.97))$ and remained significant after Bonferroni correction (corrected $P=0.043$ ). Haplotype analyses performed for SNPs in the ZEB1 and COL8A2 genes showed no significant associations (data not shown).

\section{Immunohistochemistry}

Immunohistochemistry was performed on sections of corneas from individuals with advanced FED and normal individuals to investigate differences in distribution of the CLU and TGFBI proteins. Immunolabelling results from all three FED corneas showed similar expression patterns for each protein examined. In corneal sections immunolabelled for CLU, weak CLU-positive labelling was observed in the cytoplasm in endothelial cells in normal corneas, but reduced cell numbers made it difficult to ascertain similar labelling in FED-affected corneas (Figure 1, CE, bottom panels). Weak CLU labelling was also seen in the DM in both normal and FED corneas (Figure 1, DM, bottom panels). The protein distributed solely in the stromal aspect of the thickened DM in affected corneas (Figure 1, DM, bottom left panel), whereas uniform distribution was observed in normal corneas (Figure 1, bottom right panel). Similar stromal CLU labelling was observed in both normal and FED corneas (Figure 1, S). No labelling for CLU was observed in normal corneal epithelium (Figure 1, top right panel), but strong cytoplasmic labelling was observed in the corneal epithelium in FED-affected corneas (Figure 1, Epi, top left panel).

Immunolabelling for TGFBI did not show detectable protein expression in endothelial cells either in FED-affected or normal corneas (Figure 2, CE, bottom panels). However, TGFBI expression was observed in guttae on the posterior face of the DM in FEDaffected corneas (Figure 2, DM, bottom left panel). Uniform distribution of the protein was seen in the DM in normal and affected corneas (Figure 2, DM, bottom panels). As expected, the DM was thicker in affected corneas and had guttae (Figure 2, arrow in bottom left panel). TGFBI labelling was observed in the stroma in both normal and FED corneas, but the normal stroma showed denser labelling indicating difference in intensity of expression (Figure 2, S). Interestingly, a thickened Bowman's layer devoid of TGFBI labelling was observed in FED-affected corneas (Figure 2, BL, top left panel). Comparatively, Bowman's layer was thinner and positively labelled for TGFBI in normal corneas (Figure 2, double-headed arrow, top right panel). No

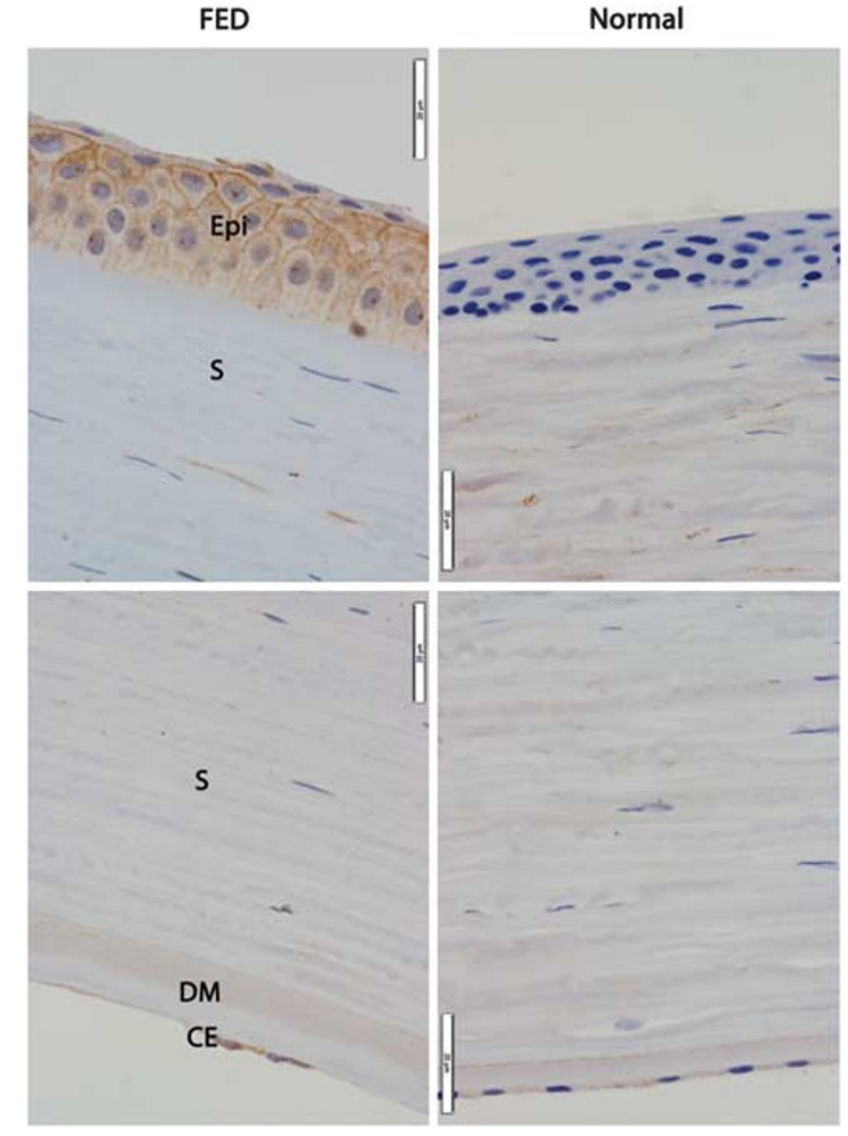

Figure 1 Immunohistochemical labelling for clusterin (CLU) protein in sections of corneas from FED (left panels) and normal (right panels) individuals. Anti-CLU labelling (brown) can be seen in FED corneal epithelium (Epi), but is absent in normal corneal epithelium. Similar distribution of CLU can be seen in the corneal stroma (S) in both FED and normal corneas. CLU is expressed only along the anterior/stromal face of the DM in FED cornea. In contrast, it is present throughout the DM in normal cornea. CLU is expressed in the CE of both FED and normal corneas. The CE in FED cornea has fewer cells compared with normal cornea, which is consistent with endothelial degeneration in FED. Magnification $\times 600$. Representative images from three experiments on independent specimens are shown.

labelling was observed in the epithelium in normal corneas (Figure 2, Epi, top right panel). In contrast, positive immunolabelling for TGFBI was observed in the cytoplasm and between adjacent epithelial cells in FED-affected corneas (Figure 2, Epi, top left panel).

\section{DISCUSSION}

The results of this study support recent studies reporting that SNPs in the TCF4 gene are strongly associated with FED, ${ }^{19,20}$ and suggest that TCF4 contributes to FED pathogenesis in Caucasian Australians. We observed that individuals with the minor allele C at rs17595731, or minor allele $\mathrm{G}$ at rs613872 in TCF4, are four times more likely to develop FED than the non-carriers. In addition, individuals with allele $\mathrm{G}$ at rs 9954153 or allele $\mathrm{T}$ at rs 2286812 have 2.6 times the risk of developing FED than non-carriers. A previous report found that all four TCF4 SNPs were independently associated with FED, ${ }^{19}$ and that a dominant model is most likely. Our data support these findings.

TCF4 encodes the E2-2 protein, which is a member of the ubiquitously expressed class I basic helix-loop-helix transcription 


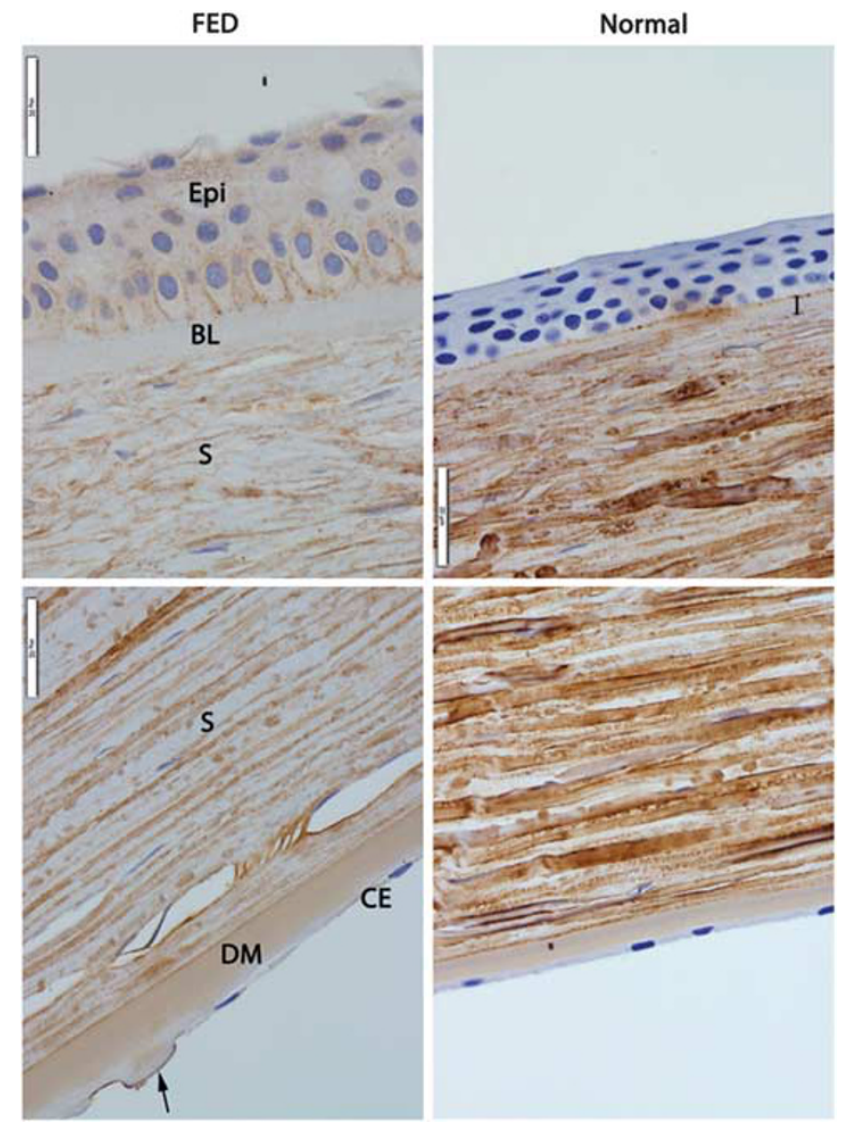

Figure 2 Immunohistochemical labelling for transforming growth factor $\beta$-induced (TGFBI) protein in sections of corneas from FED (left panels) and normal (right panels) individuals. Anti-TGFBI labelling (brown) can be seen in FED corneal epithelium (Epi), but is absent in normal corneal epithelium. TGFBI is not expressed in abnormally thicker Bowman's layer in FED cornea (BL), but is expressed in the thinner Bowman's layer in the normal cornea (double-headed arrow). Expression of TGFBI is less intense in FED corneal stroma (S) compared with normal corneal stroma. Similar distribution of TGBFI can be seen in the DM in both FED and normal corneas. DM in FED cornea is thicker than that in normal cornea, and has guttae (arrow). No TGFBI expression was observed in the corneal endothelium (CE) of both FED and normal corneas. Magnification $\times 600$. Representative images from three experiments on independent specimens are shown.

factors that are involved in cellular growth and differentiation. ${ }^{27}$ E2-2 is expressed in developing CE. ${ }^{27}$ It plays an essential role in developmental processes including EMT (epithelial-mesenchymal transition), a normal aspect of cell migration in embryogenesis and tumor-cell invasion and metastasis. ${ }^{33}$ It binds to E-box promoter sequences within target genes and either suppresses or activates tissuespecific gene transcription. ${ }^{27}$ E2-2 represses expression of cell-adhesion protein E-cadherin, thereby causing loss of cellular polarity and cell-to-cell contact and hence EMT. ZEB1 protein, which also binds to E-box promoters and is involved in EMT through E-cadherin repression, ${ }^{34}$ is upregulated by E2-2. Rare pathological mutations in ZEB1 reportedly contribute to late-onset FED in Caucasians. ${ }^{6}$ These observations suggest that the TCF4 variants associated with FED could confer the disease risk by altering the expression of ZEB1. Loss of function of a copy of E2-2 through coding mutations or genomic deletions leads to Pitt-Hopkins syndrome, ${ }^{19}$ which is not known to develop in patients with FED. In addition, TCF4 maps inside the FCD2 locus on chromosome 18q. ${ }^{35}$ This locus has been linked with the disease in multiple families, but variation in the TCF4 gene does not appear to account for the linkage signal. ${ }^{20}$

Association of rs7640737 and rs10490775 SNPs in PTPRG with FED was reported, but the result did not meet the requirements for genomewide significance. ${ }^{19}$ The study by Riazuddin and co-workers ${ }^{20}$ did not replicate the rs 10490775 SNP results. Our study detected a borderline allelic association of rs7640737 and rs10490775 with FED (Table 2). While the direction of association at SNP rs10490775 is consistent between this study and that of Baratz et al, at SNP rs7640737 the risk alleles are opposite. ${ }^{19}$ Given the inconsistent findings at this locus, we conclude that as yet there is limited evidence for a true association of SNPs from PTPRG with FED, although we acknowledge that the power of our study to detect weak associations is limited.

Analysis of tag SNPs from CLU revealed a significant association of rs17466684 with advanced disease in the Caucasian Australians (Table 2). This is the first report of genetic association of CLU with FED, although further work is required to determine the causative allele. Overexpression of CLU has been shown in FED-affected corneas. $^{22}$ Its expression is also higher in corneas from normal older individuals compared with younger individuals, ${ }^{22}$ which could be relevant for late-onset FED. Our study also shows for the first time the epithelial expression of CLU in FED-affected cornea, but not normal controls (Figure 1, Epi, top left panel), implying upregulation of the protein specifically in this cell layer in the disease state. Histologically, the advanced FED-affected cornea is characterised by thickened corneal epithelium, basal corneal epithelial oedema, occasional prominence of Bowman's layer and stromal oedema, and abnormally thickened DM with guttae on its posterior edge. ${ }^{2}$ Our study confirms these findings. Furthermore, we observe differential distribution of CLU in the DM in FED-affected corneas. CLU labelling revealed two distinct layers in the FED DM, with the posterior layer remaining unstained. This pattern was not evident in normal corneas (Figure 1, bottom right panel). Whereas anterior DM is a banded layer of collagen that is laid down before birth, posterior DM is a non-banded collagen layer, which is progressively secreted by normal CE after birth. ${ }^{36}$ In FED, endothelial cells secrete an additional banded collagen layer, posterior collagenous layer, in the extra posterior DM. ${ }^{37}$ Thus, our data may imply that CLU is expressed by corneal endothelial cells, but not secreted in FED cornea. This interpretation is consistent with previous findings, which showed overexpression of CLU in endothelial cells, but not throughout the CE+DM complex in FED cornea. ${ }^{22}$

On the basis of these findings, we hypothesise that variants in the $C L U$ gene may affect its secretion by corneal endothelial cells, exposing these cells to physiological stresses such as aging, oxidative stress and apoptosis, which may be important in FED pathogenesis. ${ }^{24,25}$ Genetic variation at the $C L U$ gene is also associated with pseudoexfoliation syndrome, a disease caused by accumulation of extracellular matrix proteins on the surface of the lens and other structures within the anterior chamber. ${ }^{38,39} \mathrm{FED}$ is also characterised by the accumulation of extracellular matrix proteins, specifically in DM. ${ }^{40}$

Genetic analysis of five tag SNPs in TGFBI did not detect any association of individual SNPs with FED, although haplotype analysis revealed that the TAAAT haplotype is significantly associated with carriers being around twice as likely to develop FED. The TAAAT haplotype is possibly tagging FED-associated variants in TGFBI not tagged by the individual SNPs. This possibility requires further followup. Immunolabelling of TGFBI revealed differential expression and distribution of the protein in FED and normal corneas. Our data show that TGFBI is upregulated in FED corneal epithelium and concentration of immunolabelling between adjacent cells suggests that the protein is secreted by stressed epithelial cells. This assumption is 
consistent with previous studies showing that injured/diseased corneal epithelial cells produce and secrete extracellular TGFBI to protect against injury. ${ }^{41}$ We also observed that the Bowman's layer in FEDaffected cornea was thicker than that in normal cornea and devoid of TGFBI staining (Figure 2). The Bowman's layer is a condensation of the outermost portion of the corneal stroma, which is composed of type I and V collagens interlaced with keratocytes. Stromal keratocytes produce TGFBI that is detectable in Bowman's layer. A recent study demonstrates that $54-63 \%$ of anterior keratocytes are depleted in $\mathrm{FED},{ }^{42}$ possibly explaining the lack of TGFBI expression in the Bowman's layer of FED cornea in our study. Consistently, our study revealed less intense stromal TGFBI expression in FED corneas, especially in the anterior stroma (Figure 2). Furthermore, TGFBI was present in guttae in FED corneas, consistent with the previous findings. ${ }^{22}$ Surprisingly, we did not detect TGFBI expression in endothelial cells in normal or FED corneas. Previous studies reported TGFBI expression and secretion by endothelial cells in normal and FED corneas, with TGFBI overexpression in the latter corneas. ${ }^{22}$ These studies used flat mounts of CE+DM complex, whereas we used sections of whole corneas. Therefore, our method would be less sensitive in identifying expression in the CE that could explain the absence of TGFBI labelling in these cells in both normal and diseased corneas.

TGFBI mediates cell adhesion by interacting with collagens, fibronectin and integrins, which are major components of the DM. ${ }^{43,44}$ TGF- $\beta$, which induces the TGFBI protein, has a reported role in the regulation of TCF4 and ZEB1 through signaling pathways in EMT. ${ }^{26}$ Mutations in TGFBI cause some human corneal dystrophies, but have not been reported to cause FED. ${ }^{2}$ Our study has revealed the first possible association of TGFBI with FED and upregulation of TGFBI in the corneal epithelium in FED. We speculate that TGFBI is overexpressed by injured corneal epithelial cells during the end stage of the disease. TGFBI is known to be expressed in corneas damaged through other mechanisms, including trauma and scarring in addition to dystrophies. ${ }^{45,46}$ Thus, it is not possible to distinguish in this study between a causative effect for TGFBI or a responsive one.

Genetic analysis of $Z E B 1$ and COL8A2 revealed no significant association of variation in these genes with FED in our study. Mutations in COL8A2 cause early-onset $\mathrm{FED}^{11}$ and mutations in ZEB1 account for a small proportion of late-onset familial FED in Caucasians. ${ }^{6}$ The majority of patients in this study had late-onset FED. Thus, a lack of association at ZEB1 and COL8A2 is not surprising. Furthermore, any variant with which these tag SNPs are in low linkage disequilibrium are not well tagged and cannot be fully assessed by this methodology. In addition, this study was sufficiently powered to detect an OR of 1.5 or above. ${ }^{31}$ A larger number of cases would be required to detect association with $\mathrm{OR}$ of $<1.5$ in any of these genes.

This study has independently replicated genetic association of TCF4 with advanced FED in Caucasian Australians, confirming that TCF4 contributes to FED risk. Our study did not support the association of the PTPRG gene with FED. However, we did find a novel although nominal association of $C L U$ and TGFBI with FED. Immunohistochemistry data revealed differential expression and distribution of the CLU and TGFBI proteins in FED corneas, supporting a role for these proteins in disease processes. On the basis of these and previous findings in the literature, we propose that aberrant adhesion, mesenchymal transition of $\mathrm{CE}$ and physiological stress contribute to FED pathogenesis.

\section{CONFLICT OF INTEREST}

The authors declare no conflict of interest.

\section{ACKNOWLEDGEMENTS}

We thank all the participants for taking part in this study. This work was supported by grant from the Ophthalmic Research Institute of Australia. KPB and JEC are, respectively, supported by the National Health and Medical Research Council of Australia Career Development Award and Practitioner Fellowship.

1 Kannabiran C: Genetics of corneal endothelial dystrophies. J Genet 2009; 88 : 487-494.

2 Klintworth G: Corneal dystrophies. Orphanet J Rare Dis 2009; 4: 7

3 Adamis AP, Filatov V, Tripathi BJ, Tripathi RC: Fuchs' endothelial dystrophy of the cornea. Surv Ophthalmol 1993; 38: 149-168.

4 Kenney MC, Labermeier U, Hinds D, Waring GO: Characterization of the Descemet's membrane/posterior collagenous layer isolated from Fuchs' endothelial dystrophy corneas. Exp Eye Res 1984; 39: 267-277.

5 Price MO, Gorovoy M, Benetz BA et al: Descemet's stripping automated endothelial keratoplasty outcomes compared with penetrating keratoplasty from the cornea donor study. Ophthalmology 2010; 117: 438-444.

6 Riazuddin SA, Zaghloul NA, Al-Saif A et al: Missense mutations in TCF8 cause lateonset Fuchs corneal dystrophy and interact with FCD4 on chromosome 9p. Am J Human Genet 2010; 86: 45-53.

7 Santo RM, Yamaguchi T, Kanai A, Okisaka S, Nakajima A: Clinical and histopathologic features of corneal dystrophies in Japan. Ophthalmology 1995; 102: 557-567.

8 Vithana EN, Morgan PE, Ramprasad V et al: SLC4A11 mutations in Fuchs endothelial corneal dystrophy. Hum Mol Genet 2008; 17: 656-666.

9 Williams KL, Bartlett MT, Kelly CM, Coster LDJ: The Australian Corneal Graft Registry: 2007 Report Adelaide: Flinders University 2007.

10 Biswas S, Munier FL, Yardley J et al: Missense mutations in COL8A2, the gene encoding the $\alpha 2$ chain of type VIII collagen, cause two forms of corneal endothelial dystrophy. Hum Mol Genet 2001; 10: 2415-2423.

11 Mok JW, Kim HS, Joo CK: Q455V mutation in COL8A2 is associated with Fuchs' corneal dystrophy in Korean patients. Eye 2008; 23: 895-903.

12 Kabosova A, Azar DT, Bannikov GA et al: Compositional differences between infant and adult human corneal basement membranes. Invest Ophthalmol Vis Sci 2007; 48: 4989-4999.

13 Weiss JS: Corneal dystrophies: molecular genetics to therapeutic intervention-Fifth ARVO/Pfizer Ophthalmics Research Institute Conference. Invest Ophthalmol Vis Sci 2010; 51: 5391-5402.

14 Mehta JS, Vithana EN, Tan DTH et al: Analysis of the posterior polymorphous corneal dystrophy 3 gene, TCF8, in late-onset Fuchs endothelial corneal dystrophy. Invest Ophthalmol Vis Sci 2008; 49: 184-188.

15 Hurt E, Saykally J, Anose B, Kalli K, Sanders M: Expression of the ZEB1 ( $\delta E F 1)$ transcription factor in human: additional insights. Mol Cell Biochem 2008; 318: 89-99.

16 Liu Y, El-Naggar S, Darling DS, Higashi Y, Dean DC: Zeb1 links epithelialmesenchymal transition and cellular senescence. Development 2008; 135: 579-588.

17 Vandewalle C, Van Roy F, Berx G: The role of the ZEB family of transcription factors in development and disease. Cell Mol Life Sci 2009; 66: 773-787.

18 Riazuddin SA, Vithana EN, Seet L-F et al: Missense mutations in the sodium borate cotransporter SLC4A11 cause late-onset Fuchs corneal dystrophya. Hum Mutat 2010; 31: 1261-1268.

19 Baratz KH, Tosakulwong N, Ryu E et al: E2-2 protein and Fuchs' corneal dystrophy. N Engl J Med 2010; 363: 1016-1024.

20 Riazuddin SA, McGlumphy EJ, Yeo WS, Wang J, Katsanis N, Gottsch JD: Replication of the TCF4 intronic variant in late-onset Fuchs corneal dystrophy and evidence of independence from the FCD2 locus. Invest Ophthalmol Vis Sci 2011; 52: 2825-2829.

21 Thalamuthu A, Khor CC, Venkataraman D et al: Association of TCF4 gene polymorphisms with Fuchs corneal dystrophy in the chinese. Invest Ophthalmol Vis Sci 2011; 52: 5573-5578.

22 Jurkunas UV, Bitar M, Rawe I: Colocalization of increased transforming growth factor\{beta\}-induced protein (TG. Invest Ophthalmol Vis Sci 2009; 50: 1129-1136.

$23 \mathrm{Kim} \mathrm{JH}, \mathrm{Kim} \mathrm{JH}$, Jun $\mathrm{HO}$ et al: Protective effect of clusterin from oxidative stressinduced apoptosis in human retinal pigment epithelial cells. Invest Ophthalmol Vis Sci 2010; 51: 561-566

24 Borderie VM, Baudrimont M, Vallee A, Ereau TL, Gray F, Laroche L: Corneal endothelial cell apoptosis in patients with Fuchs' dystrophy. Invest Ophthalmol Vis Sci 2000; 41: 2501-2505

25 Jurkunas UV, Bitar MS, Funaki T, Azizi B: Evidence of oxidative stress in the pathogenesis of Fuchs endothelial corneal dystrophy. Am J Pathol 2010; 177: 2278-2289.

26 Fuxe J, Vincent T, Garcia de Herreros A: Transcriptional crosstalk between TGF-beta and stem cell pathways in tumor cell invasion: role of EMT promoting Smad complexes. Cell Cycle 2010; 9: 2363-2374.

27 Wright AFMCP, Dhillon BBB: Major progress in Fuchs's corneal dystrophy. N Eng/ J Med 2010; 363: 1072-1075.

28 Krachmer JH, Purcell Jr JJ, Young CW, Bucher KD: Corneal endothelial dystrophy: a study of 64 families. Arch Ophthalmol 1978; 96: 2036-2039. 
29 Barrett JC, Fry B, Maller J, Daly MJ: Haploview: analysis and visualization of LD and haplotype maps. Bioinformatics 2005; 21: 263-265.

30 Purcell S, Neale B, Todd-Brown K et al: PLINK: a tool set for whole-genome association and population-based linkage analyses. Am J Hum Genet 2007; 81: 559-575.

31 Purcell S, Cherny SS, Sham PC: Genetic Power Calculator: design of linkage and association genetic mapping studies of complex traits. Bioinformatics 2003; 19: 149-150.

32 Sharma S, Chataway T, Burdon KP et al: Identification of LOXL1 protein and apolipoprotein $\mathrm{E}$ as components of surgically isolated pseudoexfoliation material by direct mass spectrometry. Exp Eye Res 2009; 89: 479-485.

33 Sobrado VR, Moreno-Bueno G, Cubillo E et al: The class I bHLH factors E2-2A and E2-2B regulate EMT. J Cell Sci 2009; 122: 1014-1024.

34 Eger A, Aigner K, Sonderegger $\mathrm{S}$ et al: DeltaEF1 is a transcriptional repressor of $\mathrm{E}$-cadherin and regulates epithelial plasticity in breast cancer cells. Oncogene 2005; 24: 2375-2385.

35 Sundin $\mathrm{OH}$, Broman KW, Chang HH, Vito ECL, Stark WJ, Gottsch JD: A common locus for late-onset Fuchs corneal dystrophy maps to 18q21.2-q21.32. Invest Ophthalmol Vis Sci 2006; 47: 3919-3926.

36 Johnson DH, Bourne WM, Campbell RJ: The ultrastructure of Descemet's membrane: I. Changes with age in normal corneas. Arch Ophthalmol 1982; 100: 1942-1947.

37 Waring GO III: Posterior collagenous layer of the cornea. Ultrastructural classification of abnormal collagenous tissue posterior to Descemet's membrane in 30 cases. Arch Ophthalmol 1982; 100: 122-134.
38 Burdon KP, Sharma S, Hewitt AW et al: Genetic analysis of the clusterin gene in pseudoexfoliation syndrome. Mol Vis 2008; 14: 1727-1736.

39 Krumbiegel $M$, Pasutto $F$, Mardin $\mathrm{CY}$ et al: Exploring functional candidate genes for genetic association in German patients with pseudoexfoliation syndrome and pseudoexfoliation glaucoma. Invest Ophthalmol Vis Sci 2009; 50: 2796-2801.

40 Wilson SE, Bourne WM: Fuchs' dystrophy. Cornea 1988; 7: 2-18.

41 Yellore VS, Rayner SA, Aldave AJ: TGFB1-induced extracellular expression of TGFBIp and inhibition of TGFBIp expression by RNA interference in a human corneal epithelial cell line. Invest Ophthalmol Vis Sci 2011; 52: 757-763.

42 Hecker LA, McLaren JW, Bachman LA, Patel SV: Anterior keratocyte depletion in Fuchs endothelial dystrophy. Arch Ophthalmol 2011; 129: 555-561.

43 Billings PC, Whitbeck JC, Adams CS et al: The transforming growth factor- $\hat{l}^{2}$. inducible matrix protein $\hat{\imath}^{2}$ ig-h3 interacts with fibronectin. J Biol Chem 2002; 277 : 28003-28009.

44 Takeyama Y, Sato M, Horio M et al: Knockdown of ZEB1, a master epithelial-tomesenchymal transition (EMT) gene, suppresses anchorage-independent cell growth of lung cancer cells. Cancer Lett 2010; 296: 216-224.

45 Aldave AJ, Principe AH, Lin DY, Yellore VS, Small KW: Lattice dystrophy-like localized amyloidosis of the cornea secondary to trichiasis. Cornea 2005; 24: $112-115$.

46 Suesskind D, Auw-Haedrich C, Schorderet DF, Munier FL, Loeffler KU: Keratoepithelin in secondary corneal amyloidosis. Graefes Arch Clin Exp Ophthalmol 2006; 244: 725-725-731. 